\title{
An Optimized SVM Using Multiple Grid Search and Particle Swarm Optimization for Medication Errors Detection
}

\author{
Aloysius Ari Wicaksono ${ }^{1}$, Antoni Wibowo ${ }^{2}$ \\ ${ }^{1}$ Bina Nusantara University, Indonesia, aloysius.wicaksono@binus.ac.id \\ ${ }^{2}$ Bina Nusantara University, Indonesia, anwibowo@ binus.edu
}

\begin{abstract}
Many medication error incidents could endanger patient safety, therefore a lot of effort to prevent medication errors were needed. Technology-based solution was also being developed to prevent medication errors. Handwritten prescriptions that were difficult to read were replaced by computer-based prescription orders. In addition, anomaly detection machine learning algorithm was also used to automatically detect prescription dosage errors. SVM was a machine learning method that can solve both linear and nonlinear problems with good performance classification accuracy rate. However, SVM performance depended on the parameter selection, such as kernel, $\mathrm{C}$, and $\gamma$. Multiple Grid Search Particle Swarm Optimization (MGSPSO) was proposed to get optimal SVM parameter. The comparative experiment on medication error detection showed that the MGSPSO provided higher accuracy rate, compared to other methods applied to this problem. In this experiment, MGSPSO could increase Grid Search and Particle Swarm Optimization (PSO) accuracy rate about 3\%. MGSPSO also could decrease Grid Search recognition time.
\end{abstract}

Key words: Anomaly Detection, Medication Error, Support Vector Machine, Grid Search, Particle Swarm Optimization.

\section{INTRODUCTION}

Medication errors were errors in the examination process that can endanger patient's health errors which occur not only due to the wrong type of drug, but also the wrong dose of the drug [1]. To prevent medical error, medication safety concept was proposed. The concept of medication safety began to become a global concern after an unexpected event in America as 98,000 people died due to medical errors (errors in medical services) and 7,000 cases due to medication error. Medication errors were the most common and most common type of medical error [2].

Several approaches had been proposed to prevent medication error through a manual approach. One of them was by training pharmacists. Pharmacists were trained to be able to detect any prescription dosage errors before the drugs were given to patients. Prescription data were reviewed by pharmacists including the name, weight, age, and gender of the patient, the dosage of the drug, the rules, and the drug reaction [3]. Knowledge about medication errors must be increased for pharmacists, for example by training on basic principles of therapy. Pharmacists also must always have the latest knowledge about the therapies and drugs used [1].

A technology approach was also developed to reduce medication error. Handwriting prescription by doctors often difficult to read [4]. It became one of the causes of errors in medication error. Therefore, the Institute of Safe Medication Practices recommended eliminating handwriting prescription. CPOE (Computerized Physician Order Entry) based Electronic Hospital Records (EHR) was used to overcome this problem [5]. As shown in figure 1, data from The Centers for Disease Control and Prevention (CDC) shown the use of EHR in the United States in 2001 as much as $18.2 \%$ increased to $78.4 \%$ in 2013 and continues to increase to $88.4 \%$ in 2017. This computerized prescription order processing proven to be able to reduce the medication error around $12.5 \%$ [6]. Technology was also used to reduce the number of prescription dosage errors. Electronic Dosing Calculator was developed to calculate exact dosage of the drug to be given to patients. This method was claimed to be able to eliminate any prescription errors for known drugs. However, this system had weaknesses, that is, the formula depends very much on the team of doctors where the program is implemented. In addition, not all errors could be identified and recorded [7]. Machine learning was also used to prevent medication error. DDC-Outlier (Density-Distance-Centrality) method was developed using parameters of drug type, dosage, and frequency. This method gave F-Measure result of 0.68. However, the test did not use the patient's weight parameters so that the detection of dose errors was not really specific to the patient's needs [8].

SVM (Support Vector Machine) was a machine learning method that can solve both linear and nonlinear problems. SVM had good performance classification accuracy rate [9]. SVM used in many classification problem, such as credit scoring [10], intrusion detection [11], learning cancer genomics [12], image classification [13], lung cancer detection [14], detection of false agricultural insurance claims [15] and many more. 
However, SVM performance depended on the parameter selection, such as kernel, $\mathrm{C}$, and $\gamma$. There are some kernel options in SVM such as linear, poly, rbf [16].

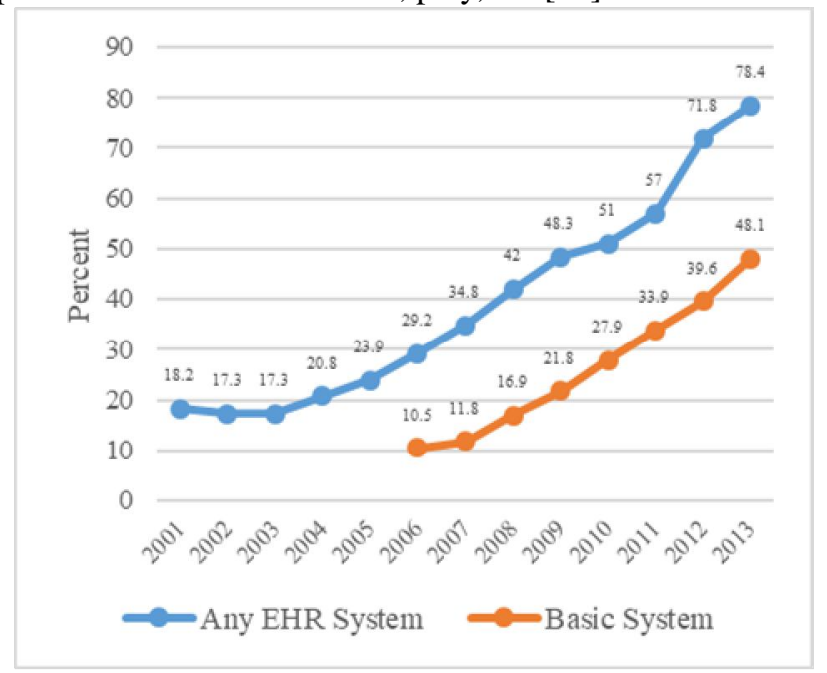

Figure 1: Increasing Use of EHR in United States

In this paper, an optimized SVM method using PSO combined with multiple Grid Search method has been proposed. Best kernel, $\mathrm{C}$, and $\gamma$ parameter are selected using this method.

\section{OPTIMIZED SVM METHOD}

There are several method to get the optimal SVM parameter, such as:

Grid Search: Grid search is exhaustive search method. This method will evaluate best fitness value from each given parameter. Grid search used for optimized SVM use kernel, C and $\gamma$ parameter. In this method, all kernel, $\mathrm{C}$ and $\gamma$ combination were evaluated to get highest accuracy. This method has good accuracy. However, this method was very slow when evaluated many parameter combinations $[17,18]$. Bilinear Grid Search Method (BGSM) was proposed to improve grid search performance. Grid search was performed twice. First, large range value of $\mathrm{C}$ and $\gamma$ were used to get optimal parameter. Then, grid search was performed again with more detailed combination of $\mathrm{C}$ and $\gamma$ based on $\mathrm{C}$ and $\gamma$ got from first step to get optimal $\mathrm{C}$ and $\gamma$ parameter [19].

Particle Swarm Optimization: Particle Swarm Optimization was a population optimization technique proposed by Kennedy and Eberhart [20, 21]. PSO was inspired by flocks of birds that migrate to a place. In this algorithm, there were several particles in an n-dimensional space. Each particle had a fitness value that will be evaluated using fitness function. When a particle with the best fitness value was found, all particles would move in the direction of the best particle. The particles would be evaluated continuously according to the number of iterations. For each iteration, the particle position would be updated according to the previous best position. PSO was easy to implement and can be used in many optimization problem, such as for feature selection problem $[22,23,24]$ or finding optimal model parameter [25, 26, 27].
However, this algorithm was easy to fall into local optimum when implemented using wide range [28].

Grid Search and Particle Swarm Optimization Combination: Xiao et al proposed combination Grid Search and Particle Swarm Optimization (GSPSO) to get optimal C and $\gamma$ for SVM. The basic idea from this research was grid search with rbf kernel was used to get optimal $\mathrm{C}$ and $\gamma$ value from large range combination of $\mathrm{C}$ and $\gamma$ value. Then, PSO method would be performed for more detailed search to get optimal parameter combinations $(\mathrm{C}, \gamma)$ using specific range value based on $\mathrm{C}$ and $\gamma$ value from first step. This research gave better result compare to grid search alone and PSO alone [29]. However, this research only use rbf kernel without compare to other kernel performance. Besides that, range value used for PSO operation was not mentioned.

\section{PROPOSED METHOD}

In this study, combination multiple grid search and particle swarm optimization (MGSPSO) will be used for optimized SVM. Precision, recall, and F-measure from this method will be compared with grid search (GS) method alone, particle swarm optimization (PSO) method alone, bilinear grid search method (BGSM) and grid search particle swarm optimization (GSPSO). F-measure value will be evaluated to get best optimized SVM because F-measure value include accuracy, precision, and recall. F-measure value will be calculated using formula as mentioned in figure 2 .

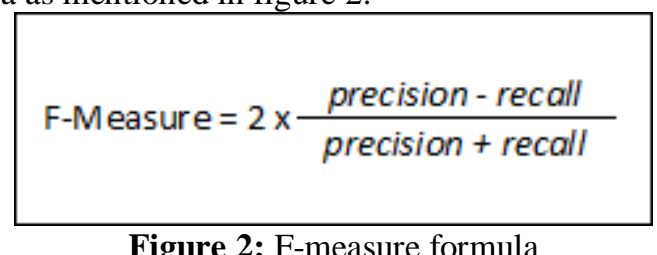

Optimized SVM using MGSPSO is explained in figure 3. First, Grid Search will be used to get the optimal kernel, C, and $\gamma$ parameter with a large range combination of $\mathrm{C}$ and $\gamma$ value. The range of $\mathrm{C}$ value used for this study is [0.001, 0.01 , $1,100,1000]$. The range of $\gamma$ value used for this study is $[0.001,0.01,1,100,1000]$. Kernel value used for this study is [linear, sigmoid, rbf, poly]. From these range, there are 100 combination compared for grid search operation. Best fitness value will be selected as best parameter value.

Best kernel, C, and $\gamma$ parameter will be saved as kernel1, C1, and $\gamma 1$. After that, remove best each $\mathrm{C}$ and $\gamma$ value from grid search result. Remove all kernel except kernel1 from grid search result. Then, find best grid search result from remaining parameter result. Best $\mathrm{C}$ and $\gamma$ parameter will be saved as $\mathrm{C} 2$ and $\gamma 2$. Second optimal parameter will be found from this operation.

After get $\mathrm{C} 1, \gamma 1$, kernel1, $\mathrm{C} 2$, and $\gamma 2$ value, do particle swarm operation with kernel1 as kernel value, range $\mathrm{C}$ from $\mathrm{C} 1$ and $\mathrm{C} 2$, and range $\gamma$ from $\gamma 1$ and $\gamma 2$. Fitness value will be calculated using cross validation with 5 fold. Optimal kernel, 
$\mathrm{C}$, and $\gamma$ combination from this operation will be selected as best parameter.

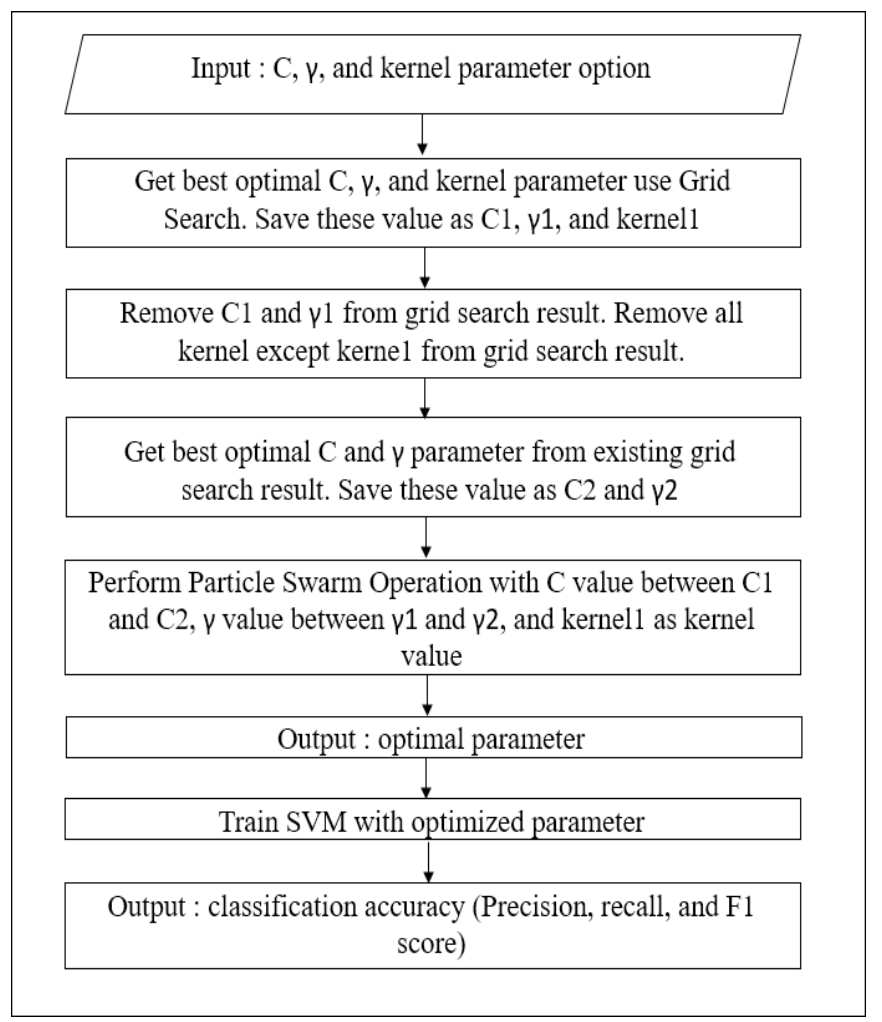

Figure 3: Optimized SVM using MGSPSO

\section{ANALYSIS RESULT}

\subsection{Dataset and Parameter}

This study use prescription dose dataset from a pediatrician clinic in Jakarta from 2016-2019. There are total 266.132 record. This dataset contains patient age, patient weight, patient height, patient temperature, item id, item dose, item unit, compound quantity, compound unit, and frequency. There are 3 drugs will be used for this research as mentioned in table 1 .

Table 1: Prescription Dataset

\begin{tabular}{|r|r|r|r|}
\hline Item ID & \multicolumn{1}{|c|}{ Dose } & \multicolumn{1}{c|}{$\begin{array}{c}\text { Data } \\
\text { Normal }\end{array}$} & $\begin{array}{c}\text { Data } \\
\text { Anomaly }\end{array}$ \\
\hline 398 & 4 & 533 & 31 \\
\hline 640 & 250 & 563 & 24 \\
\hline 1112 & 5 & 1031 & 150 \\
\hline
\end{tabular}

\subsection{Preprocessing}

Preprocessing data must be performed to ensure data quality. There are several preprocessing task describe below:

Compound Unit: Some prescribed medications are presented in drug dose unit and others in drug unit. To avoid mistakes in the quantity, all prescription use dose unit. Quantity prescription are recalculated using drug dose for non-standard units.

Frequency: Each prescription has frequency in different format, such as daily, hourly, and weekly. This data will be standardized using daily frequency.

Oversampling: Oversampling will be performed to prevent imbalance data problem. This step will be done using the SMOTE method. After SMOTE operation, the number of data increased as shown in Table 2.

Table 2: Prescription Dataset after SMOTE operation

\begin{tabular}{|r|r|r|}
\hline Item ID & Before SMOTE & After SMOTE \\
\hline 398 & 564 & 1066 \\
\hline 640 & 587 & 1126 \\
\hline 1112 & 1181 & 2146 \\
\hline
\end{tabular}

\subsection{Performance Result}

Table 3 show precision result of each method using 3 different dataset. MGSPSO has higher precision result than both PSO and Grid Search. Table 4 show recall result of each method. GSPSO method gives higher recall value than other method. In general, as shown in table 4, MGSPSO has the higher overall accuracy than the PSO and the Grid Search.

Table 3: Precision comparison

\begin{tabular}{|l|r|r|r|}
\hline Algorithm & $\mathbf{3 9 8}$ & $\mathbf{6 4 0}$ & $\mathbf{1 1 1 2}$ \\
\hline GS & 0.8768116 & 0.8768116 & 0.9475524 \\
\hline PSO & 0.9242424 & 0.8832117 & 0.9611307 \\
\hline BGSM & 0.9844961 & 0.884058 & 0.9575972 \\
\hline GSPSO & 0.9844961 & 0.8785714 & 0.9542254 \\
\hline MGSPSO & $\mathbf{1}$ & $\mathbf{0 . 9 1 6 6 6 7}$ & $\mathbf{0 . 9 7 1 2 2 3}$ \\
\hline
\end{tabular}

Table 4: Recall comparison

\begin{tabular}{|l|r|r|r|}
\hline Algorithm & $\mathbf{3 9 8}$ & $\mathbf{6 4 0}$ & $\mathbf{1 1 1 2}$ \\
\hline GS & 0.9837398 & 0.9837398 & 0.992674 \\
\hline PSO & 0.953125 & 0.9837398 & 0.996337 \\
\hline BGSM & 0.9921875 & 0.9918699 & 0.992674 \\
\hline GSPSO & $\mathbf{0 . 9 9 2 1 8 7 5}$ & $\mathbf{1}$ & $\mathbf{0 . 9 9 2 6 7 4}$ \\
\hline MGSPSO & 0.984375 & 0.98374 & 0.989011 \\
\hline
\end{tabular}

Table 5: F-Measure comparison

\begin{tabular}{|l|r|r|r|}
\hline Algorithm & $\mathbf{3 9 8}$ & $\mathbf{6 4 0}$ & $\mathbf{1 1 1 2}$ \\
\hline GS & 0.9272031 & 0.9272031 & 0.9695886 \\
\hline PSO & 0.9384615 & 0.9307692 & 0.9784173 \\
\hline BGSM & 0.9883268 & 0.9348659 & 0.9748201 \\
\hline GSPSO & 0.9883268 & 0.9353612 & 0.97307 \\
\hline MGSPSO & $\mathbf{0 . 9 9 2 1 2 6}$ & $\mathbf{0 . 9 4 9 0 2}$ & $\mathbf{0 . 9 8 0 0 3 6}$ \\
\hline
\end{tabular}

Table 7 shows the recognition time of each method with different dataset. The results show that recognition time of the PSO is shorter than proposed method. However, the recognition time of the MGSPSO is shorter than the Grid Search method. 
Table 6: Mean Performance comparison

\begin{tabular}{|l|c|c|r|}
\hline Algorithm & Precision & Recall & \multicolumn{1}{c|}{ F-Measure } \\
\hline GS & 0.9003919 & 0.9867179 & 0.9413316 \\
\hline PSO & 0.9228616 & 0.9777339 & 0.949216 \\
\hline BGSM & 0.9420504 & 0.9922438 & 0.9660043 \\
\hline GSPSO & 0.9390976 & $\mathbf{0 . 9 9 4 9 5 3 8}$ & 0.965586 \\
\hline MGSPSO & $\mathbf{0 . 9 6 2 6 2 9 9}$ & 0.9857086 & $\mathbf{0 . 9 7 3 7 2 7 3}$ \\
\hline
\end{tabular}

Table 7: Time comparison

\begin{tabular}{|l|r|r|r|}
\hline Algorithm & $\mathbf{3 9 8}$ & $\mathbf{6 4 0}$ & $\mathbf{1 1 1 2}$ \\
\hline GS & 10.2 & 10.3 & 15.6 \\
\hline PSO & $\mathbf{5 . 2}$ & $\mathbf{5 . 4}$ & $\mathbf{8 . 1}$ \\
\hline BGSM & 12 & 12.4 & 17.2 \\
\hline GSPSO & 8.4 & 8.2 & 11.6 \\
\hline MGSPSO & 8.3 & 8.4 & 11.5 \\
\hline
\end{tabular}

\subsection{Discussion}

The results showed that MGSPSO had the higher accuracy than the PSO and the Grid Search. PSO had better accuracy than grid search because PSO had wider range value compared to Grid Search. However, when use high-dimensional space, PSO algorithm was easy to fall into local optimum. PSO could give higher accuracy when use smaller dimensional space. To decrease PSO range value, MGSPSO used best and second best grid search result. MGSPSO also had higher accuracy than Grid Search because like PSO, it also had wider range value compared to Grid Search.

PSO gave best recognition time because the number of SVM parameter combinations compared by PSO method was less than other method. MGSPSO could decrease Grid Search recognition time when combine with PSO. Grid search only used for large step combination of $\mathrm{C}$ and $\gamma$. Then, PSO was performed for more detail combination.

\section{CONCLUSION}

Although SVM worked well with default value, SVM performance could be improved significantly using parameter optimization. One of the biggest problems of SVM parameter optimization was there was no exact ranges of $\mathrm{C}$ and $\gamma$ values. Optimized SVM using Grid Search was very powerful and it was able to improve the accuracy significantly. However, Grid Search method had several disadvantages, it was extremely slow and furthermore it may lead to very long execution time. Particle Swarm Optimization also could improve SVM accuracy. However, this method was easy to fall into local optimum when implemented using wide range.

Multiple Grid Search and Particle Swarm Optimization (MGSPSO) had been developed to improve SVM accuracy. When tested using prescription dataset, MGSPSO provided higher accuracy rate, compared to other methods applied to this problem. This method could be used for better medications error detection. It could increase Grid Search and Particle Swarm Optimization (PSO) accuracy rate about 3\%.

However, this study had not used the optimal PSO parameters. Adaptive PSO parameter can be one of the major future works.

\section{REFERENCES}

[1] J. K. Aronson, "Medication errors: definitions and classification," British Journal of Clinical Pharmacology, 2009.

[2] L. Kohn, J. M. Corrigan and M. S. Donaldson, "To Err is Human: Building a Safer Health System," Institute of Medicine (US) Committee on Quality of Health Care in America, 2000.

[3] Departemen Kesehatan Republik Indonesia, "Keputusan Menteri Kesehatan Republik Indonesia Nomor 35 tahun 2014. Tentang standar pelayanan kefarmasian di Apotek. Departemen Kesehatan Republik Indonesia. Jakarta.," 2014.

[4] R. A. Tariq and Y. Scherbak, "Medication Errors.," in StatPearls Publishing, 2019.

[5] D. C. Radley, M. R. Wasserman, L. E. Olsho, S. J. Shoemaker, M. D. Spranca and B. Bradshaw, "Reduction in MEs in hospitals due to adoption of computerized provider order entry systems," Journal of the American Medical Informatics Association, 2013.

[6] "The Centers for Disease Control and Prevention," 2017. [Online]. Available: https://www.cdc.gov/nchs/data/nehrs/2017_NEHRS_We b_Table_EHR_State.pdf.

[7] B. Murray, M. Hilliard, M. J. Streitz and J. K. Maddry, "Evaluation of an Electronic Dosing Calculator to Reduce Pediatric Medication Errors," Clinical Pediatrics, 2019.

[8] H. D. dos Santos, A. H. D. Ulbrich, V. Woloszyn and R. Vieira, "DDC-Outlier: Preventing medication errors using unsupervised learning," IEEE Journal of Biomedical and Health Informatics, 2018. https://doi.org/10.1109/JBHI.2018.2828028

[9] P.-N. Tan, M. Steinbach, A. Karpatne and V. Kumar, Introduction to Data Mining, 2006.

[10] F.-L. Chen and F.-C. Li, "Combination of feature selection approaches with SVM in credit scoring," in Expert Systems with Applications, 2010.

[11] J. Jha and L. Ragha, "Intrusion Detection System using Support Vector Machine," International Journal of Applied Information Systems, 2013.

[12] S. Huang, N. Cai, P. P. Pacheco, S. Narandes, Y. Wang and W. Xu, "Applications of Support Vector Machine (SVM) Learning in Cancer Genomics," Cancer Genomics Proteomics, 2018.

[13] T. H. Le, H. S. Tran and T. T. Nguyen, "Applying Multi Support Vector Machine for Flower Image 
Aloysius Ari Wicaksono et al., International Journal of Emerging Trends in Engineering Research, 8(7), July 2020, 4056 - 4060

Classification," International Conference on Context-Aware Systems and Applications, 2012.

[14] D. D. Nagajyothi, R. Addagudi, T. Gunda and S. S. Logitla, "Detection of Lung Cancer using SVM Classifier," International Journal of Emerging Trends in Engineering Research, vol. 8, 2020. https://doi.org/10.30534/ijeter/2020/113852020

[15] R. Budihal and D. G. Komarswamy, "Decision Support System for Detection of False Agricultural," International Journal of Emerging Trends in Engineering Research, vol. 8, 2020. https://doi.org/10.30534/ijeter/2020/33852020

[16] H. M. Asraf, M. T. Nooritawati and M. S. Rizam, "A Comparative Study in Kernel-Based Support Vector Machine of Oil," International Symposium on Robotics and Intelligent Sensors, 2012.

[17] F. Friedrichs and C. Igel, "Evolutionary tuning of multiple SVM parameters," Neurocomputing 64, 2005.

[18] C.-W. Hsu, C.-C. Chang and C.-J. Lin, "A Practical Guide to Support Vector Classi," Department of Computer Science and Information Engineering, National Taiwai University, 2003.

[19] L. Lin, Z. Xiaolong, Z. Kai and L. Jun, "Bilinear Grid Search Strategy Based Support Vector Machines," Informatica, 2014.

[20] R. Eberhart and J. Kennedy, "A new optimizer using particle swarm theory," MHS'95. Proceedings of the Sixth International Symposium on Micro Machine and Human Science, 1995.

[21] J. Kennedy and R. Eberhart, "Particle swarm optimization," IEEE international conference on neural networks, 1995.

[22] K. Mistry, L. Zhang, S. C. Neoh, C. P. Lim and B. Fielding, "A Micro-GA Embedded PSO Feature Selection Approach to Intelligent Facial Emotion Recognition," IEEE Transactions on Cybernetics, vol. 47, 2017.

[23] C.-J. Tu, L.-Y. Chuang, J.-Y. Chang and C.-H. Yang, "Feature Selection using PSO-SVM," International Journal of Computer Science, 2007.

[24] M. Amoozegar and B. Minaei-Bidgoli, "Optimizing Multi-objective PSO based feature selection method using," Expert Systems With Applications, 2018.

[25] S. Yamada and K. Neshatian, "Hyper-parameter search in support vector machines using PSO with cellular fitness approximation," 2017 IEEE Symposium Series on Computational Intelligence, 2017.

[26] Y. Wang, H. Zhang and G. Zhang, "cPSO-CNN: An efficient PSO-based algorithm for fine-tuning hyper-parameters of convolutional neural networks," Swarm and Evolutionary Computation, vol. 49, pp. 114-123, 2019.

[27] P. R. Lorenzo, J. Nalepa, M. Kawulok, L. S. Ramos and J. R. Pastor, "Particle swarm optimization for hyper-parameter selection in deep neural networks," in GECCO '17: Proceedings of the Genetic and
Evolutionary Computation Conference, 2017. https://doi.org/10.1145/3071178.3071208

[28] M. Li, W. Du and F. Nian, "An Adaptive Particle Swarm Optimization Algorithm Based on Directed Weighted Complex Network," Sensor/Actuator Networks and Networked Control Systems, 2014. https://doi.org/10.1155/2014/434972

[29] T. Xiao, D. Ren, S. Lei, J. Zhang and X. Liu, "Based on Grid-Search and PSO Parameter Optimization," in Proceeding of the 11th World Congress on Intelligent Control and Automation, Shenyang, China, 2014. 